\title{
Absolute declinations from astrolabe data
}

\author{
V.A.F. Martin ${ }^{1,2}$ and N.V. Leister ${ }^{1}$ \\ 1 Instituto Astronômico e Geofísico, Universidade de São Paulo, CP 9638, 01065-970 São Paulo, SP, Brazil \\ 2 Observatoire de la Côte d'Azur/C.E.R.G.A., Avenue Copernic, F-06130 Grasse, France
}

Received September 24, 1996; accepted January 3, 1997

\begin{abstract}
We show the results of an evaluation of a global reduction method for Danjon astrolabe observations. The approach is much simpler but more precise than the conventional method. The new form of the equations gives us the possibility of using the entire set of an observational programme using the data from different catalogues taken at two zenith distances $\left(30^{\circ}\right.$ and $\left.45^{\circ}\right)$. The observational programme contains about 15000 observations of 381 different stars taken at the "Observatório Abrahão de Moraes" (OAM, $\phi=23^{\circ} 00^{\prime} 06^{\prime \prime} \mathrm{S} ; \lambda=03^{\mathrm{h}} 07^{\mathrm{m}} 52.2^{\mathrm{s}} \mathrm{W}$ ), Valinhos, São Paulo, Brazil. The reduction was based on the FK5 system. We discuss the possibility to compute absolute declinations through the stars belonging simultaneously to the $30^{\circ}$ and $45^{\circ}$ zenith distance programmes. The absolute declination corrections were determined for 269 stars with a precision of $0.13^{\prime \prime}$, and the value of the determined equator correction is $0.028^{\prime \prime} \pm 0.034^{\prime \prime}$. The instrumental effects were also determined.
\end{abstract}

Key words: astrometry — astrolabe — reference system — fundamental stars and other objects

\section{Introduction}

One of the intrinsic difficulties of the equal altitude method is that it is impossible to compute the absolute declinations of stars. The impossibility of fixing the equator and equinox is due to the zenith distance variations in the course of an observational night caused by colour and magnitude effects.

The classical instruments for absolute declination observations are the vertical and meridian circles. However, the difficulties encountered for obtaining absolute declinations with these instruments are well known (Podobed 1965; Fricke 1978).

A different way to determine absolute declinations is possible by the compilation of the observations at two zenith distances using a modified Danjon astrolabe.

Send offprint requests to: V.A.F. Martin
Many experiments were done with the goal of determining absolute declinations with this instrument (Krejnin 1968; Débarbat \& Guinot 1970; Krejnin 1986).

The instrument at the "Observatório Abrahão de Moraes" (OAM, $\phi=23^{\circ} 00^{\prime} 06^{\prime \prime} \mathrm{S} ; \quad \lambda=03^{\mathrm{h}} 07^{\mathrm{m}} 52.2^{\mathrm{s}} \mathrm{W}$, Valinhos, São Paulo, Brazil) is one of the few instruments in its class able to observe at two zenith distances with a simple change of prism, which enables it to determine absolute declinations of stars observed at $30^{\circ}$ and $45^{\circ}$ zenith distances. The observational programme started at 1982 at two zenith distances includes not only stars, but also the Sun and the planets. The importance of the latter is to get the orientation of the reference fundamental system (Poppe et al. 1996).

The stars are arranged in different observational programmes, in groups of fixed composition.

In the period 1974-1986, about 15000 observations were taken in the declination zone $-68^{\circ}$ to $+22^{\circ}$, thus including a significant declination zone in the southern hemisphere. The observations belong to the VL1 (Valinhos $1\left(30^{\circ}\right)$ - (Clauzet 1983), VL2 (Valinhos $2\left(30^{\circ}\right)$ - Clauzet \& Benevides-Soares 1985) and VL3 (Valinhos $3\left(45^{\circ}\right)$ Clauzet 1989) catalogues. There are about 199 stars in VL1, 126 in VL2 and 109 in VL3, for a total of 434 stars. Include in this total are 125 stars at maximum digression condition and 40 stars that are common to the three catalogues. With this data set, it was possible to determine 269 absolute declinations $\left(\delta_{\mathrm{abs}}\right)$. Although a one-to-one relation is not possible, our method allows one to obtain the declination corrections of all stars.

The real possibility of absolute declination determination was shown by Clauzet (1987). We repeated the problem making a wide analysis of the systematic effects of colour and magnitude that affect astrolabe observations (Chollet \& Sanchez 1990; Basso 1991), determining a colour-magnitude function for the modified astrolabe at Valinhos (OAM) (Martin \& Clauzet 1990; Basso 1991).

The first method of obtaining absolute declinations is due Krejnin $(1968,1986)$, but the difficulty of this application is that it restricts the observations to two zenith distances, which introduces a limitation in Krejnin's method. 
In this paper, we consider all the programme stars in a global reduction method. We accomplished the global reduction due the small number of common stars in the catalogues observed at two zenith distances. Despite the small number of stars, this method allowed a better evaluation of the observational set. We obtained a better definition of the errors involved in the process and the global definition of the colour and magnitude equations, beyond getting the equator correction $(\eta)$ and the declination corrections $(\Delta \delta)$ directly.

\section{Reduction method}

In this section, we discuss three ways to determine the absolute declination corrections: Krejnin's Method, the Method of Differences, and the Global Reduction from astrolabe data.

\subsection{Krejnin's method}

Considering all the star observations $(i)$ in the involved catalogues $(j)$, we can write the following equations for each transit (east and west - E and W):

$$
\begin{aligned}
-R_{i \mathrm{E}}^{j}= & -\Delta \alpha_{i}\left|\sin Z_{i \mathrm{E}}^{j}\right| \cos \phi+\Delta \delta_{i} \cos S_{i}^{j}+ \\
& +\xi^{j} \sin Z_{i \mathrm{E}}^{j}+\eta^{j} \cos Z_{i \mathrm{E}}^{j}-\zeta^{j} \\
-R_{i \mathrm{~W}}^{j}= & +\Delta \alpha_{i}\left|\sin Z_{i \mathrm{~W}}^{j}\right| \cos \phi+\Delta \delta_{i} \cos S_{i}^{j}+ \\
& -\xi^{j}\left|\sin Z_{i \mathrm{~W}}^{j}\right|+\eta^{j} \cos Z_{i \mathrm{~W}}^{j}-\zeta^{j}
\end{aligned}
$$

where:

$R_{\mathrm{E}}$ and $R_{\mathrm{W}}$ are the east and west mean residuals in zenith distance after addition of group corrections for east and west transits of the star (homogenisation of the stellar groups), and $S$ is the parallactic angle. The constant $\eta$ can be understood as the difference between the real and observed values of the latitude after the homogenisation of the stellar groups (related with the equator correction); the constant $\zeta$ is related to the true zenith distance of observation (instrumental effects); $Z$ is the star azimuth; $\phi$ the local latitude, and $\xi$ the constant related to the equinox correction.

Adding the mean residuals $\left(R_{\mathrm{E}}\right.$ and $\left.R_{\mathrm{W}}\right)$ above, the stellar declination corrections can be computed from astrolabe observations by the formula (Débarbat \& Guinot 1970):

$$
\begin{aligned}
\Delta \delta_{i}= & -\left[\left(R_{\mathrm{E}}+R_{\mathrm{W}}\right)\right]_{i}^{j} /\left(2 \cos S_{i}^{j}\right)-\eta^{j} \cos Z_{i}^{j} / \cos S_{i}^{j}+ \\
& +\zeta^{j} / \cos S_{i}^{j},
\end{aligned}
$$

Using the stars at maximum digression condition $(\cos S \approx 0)$, the Eq. (3) can be rewritten as a function of only the constant $\eta$ :

$$
\begin{aligned}
\Delta \delta_{i}= & {\left[\left(R_{\mathrm{E}}+R_{\mathrm{W}}\right)-2 C\right]_{i}^{j} /\left(-2 \cdot \cos S_{i}^{j}\right)+} \\
& +\eta^{j} \cdot\left(\cos \delta_{i} \cdot \sec z\right) / \cos \phi
\end{aligned}
$$

where $C$ is a constant independent of the star coordinates $\left(C=f\left(I_{i}, m_{i}\right), I_{i}=\right.$ visual colour index $(B-V)$ and $m_{i}=$ visual apparent magnitude), and $z$ is the zenith distance of observation. The possibility of computing absolute declinations rests in the determination of $\eta$.

Let $\Delta \delta_{A i}$ and $\Delta \delta_{B i}$ be the declination correction values of a given star $(i)$ observed at the zenith distances $z_{A}\left(30^{\circ}\right)$ and $z_{B}\left(45^{\circ}\right)$ at the same latitude $\phi$.

From Eq. (4), we can write:

$\begin{aligned} \Delta \delta_{A i} & =\Delta \delta_{A i}^{\prime}+\left(\eta_{A} \cdot \cos \delta_{i} \cdot \sec z_{A}\right) / \cos \phi \\ \Delta \delta_{B i} & =\Delta \delta_{B i}^{\prime}+\left(\eta_{B} \cdot \cos \delta_{i} \cdot \sec z_{B}\right) / \cos \phi\end{aligned}$

where:

$\begin{aligned} \Delta \delta_{A i}^{\prime} & =\left[\left(R_{\mathrm{E}}+R_{\mathrm{W}}\right)-2 C\right]_{i}^{A} /\left(-2 \cdot \cos S_{i}^{A}\right) \\ \Delta \delta_{B i}^{\prime} & =\left[\left(R_{\mathrm{E}}+R_{\mathrm{W}}\right)-2 C\right]_{i}^{B} /\left(-2 \cdot \cos S_{i}^{B}\right)\end{aligned}$

Therefore:

$$
\begin{aligned}
\Delta \delta_{A i} & =\Delta \delta_{B i}= \\
& =\Delta \delta_{A i}^{\prime}+\left(\eta_{A} \cdot \cos \delta_{i} \cdot \sec z_{A}\right) / \cos \phi= \\
& =\Delta \delta_{B i}^{\prime}+\left(\eta_{B} \cdot \cos \delta_{i} \cdot \sec z_{B}\right) / \cos \phi
\end{aligned}
$$

or

$\eta_{A} \cdot \sec z_{A}-\eta_{B} \cdot \sec z_{B}=\left(\Delta \delta_{B i}^{\prime}-\Delta \delta_{A i}^{\prime}\right) \cdot \cos \phi \cdot \sec \delta_{i}$.

The left-hand side is independent of the selected star and so must be the right-hand side. Therefore, we are authorised to take the average of the data from all available stars, so that we arrive at a single equation connecting $\eta_{A}$ and $\eta_{B}$.

On the other hand, the instantaneous value of the latitude $\phi$ can be written from the computed values $\phi_{A}$ and $\phi_{B}$, after application of group corrections, as:

$\phi=\overline{\phi_{A}}+\eta_{A}=\overline{\phi_{B}}+\eta_{B}$
or
$\eta_{A}-\eta_{B}=<\phi_{B}-\phi_{A}>$.

In a similar way as we have done for Eq. (5), the righthand side of Eq. (6) can be averaged along the observing time span. We thus get an independent relationship which allows the determination of $\eta_{A}$ and $\eta_{B}$ and, consequently through Eq. (4), of the star declination corrections. This is the principle of Krejnin's method. 


\subsection{Method of differences}

We reduced Eq. (3) by least squares method only for the stars common to the programmes taken at $30^{\circ}(A)$ and $45^{\circ}(B)$ zenith distances, i.e., the stars common to the VL1 and VL3 catalogues and the stars common to the VL2 and VL3 catalogues.

However, as the programmes were taken in the same declination zone and were referred to the same fundamental catalogue (FK5), we can hope that $\eta$ and $\zeta$ are small and similar, otherwise the system has a singularity.

In this case, $\eta_{\mathrm{VL} 1}=\eta_{\mathrm{VL} 3} ; \zeta_{\mathrm{VL} 1}=\zeta_{\mathrm{VL} 3} ; \eta_{\mathrm{VL} 2}=\eta_{\mathrm{VL} 3}$ and $\zeta_{\mathrm{VL} 2}=\zeta_{\mathrm{VL} 3}$, which were confirmed by the results from Krejnin's method.

Thus, we can write for each star common to both catalogues $(A$ and $B)$ :

$\rho_{A i} \cdot \cos S_{A i}=+\Delta \delta_{i} \cdot \cos S_{A i}+\eta \cdot \cos Z_{A i}-\zeta ;$

$\rho_{B i} \cdot \cos S_{B i}=+\Delta \delta_{i} \cdot \cos S_{B i}+\eta \cdot \cos Z_{B i}-\zeta$,

where: $\rho=\left(R_{\mathrm{E}}+R_{\mathrm{W}}\right) /(-2 \cdot \cos S)$ and $\Delta \delta$ is the declination correction.

It is important to note that the zenith distance correction $(\zeta)$ contains colour and magnitude effects.

From the difference between the Eqs. (7), we can write:

$$
\begin{aligned}
-\left(\rho_{A i}-\rho_{B i}\right)= & \eta\left(\cos Z_{A i} / \cos S_{A i}-\cos Z_{B i} / \cos S_{B i}\right) \\
& +\zeta\left(1 / \cos S_{B i}-1 / \cos S_{A i}\right) .
\end{aligned}
$$

The advantages of an analysis of the system of Eqs. (7) are to obtain by the least squares method the terms $\eta$ and $\zeta$, the absolute declination corrections $(\Delta \delta)$, and a better estimate of the correlation between the unknowns.

\subsection{Global reduction}

We used global reduction to determine the absolute declinations with a modern method of astrometric reduction that includes all of the information in a set of equations, with all of the unknowns.

In this method, the data and the unknowns are treated as symmetrically as possible with a determined statistical weighting. The symmetric form to use the data is directly to get the mean residuals for which it is reasonable to claim statistical independence and uniform variance.

An example of this application is the overlap reduction (Eichhorn 1960) and global reduction techniques (Benevides-Soares \& Clauzet 1986; Basso 1991; Benevides-Soares \& Teixeira 1992; Chollet \& Najid 1992; Chollet 1993; Najid 1993).

From Eq. (3), we can write:

$$
\left[\left(R_{\mathrm{E}}+R_{\mathrm{W}}\right) / 2\right]_{i}^{j}=-\Delta \delta_{i} \cos S_{i}^{j}-\eta^{j} \cos Z_{i}^{j}+\zeta^{j} .
$$

Considering all of the star observations with double passage $(\mathrm{E} / \mathrm{W})$, and the colour index and magnitude func- tion $C\left(I_{i}, m_{i}\right)^{1}$, we get the average of the mean residuals $\left(\rho_{i}^{j}\right)$ :

$$
\begin{aligned}
\rho_{i}^{j}= & {\left[\left(R_{\mathrm{E}}+R_{\mathrm{W}}\right) / 2\right]_{i}^{j}=-\Delta \delta_{i} \cos S_{i}^{j} } \\
& +\left(B_{1} m_{i}+B_{2} m_{i}^{2}+A_{1} I_{i}+\Delta z\right)^{j}-\eta^{j} \cos Z_{i}^{j}
\end{aligned}
$$

where: $B_{1}, B_{2}, A_{1}=$ magnitude and colour index coefficients; $m_{i}=$ visual apparent magnitude of the star; $I_{i}=$ visual colour index $(B-V)$ of the star and $\Delta z=$ zenith distance correction.

Equation (9) can be applied to all stars. For those which are at maximum digression condition $\left(\left|\cos S_{i}^{j}\right| \leq 0.3\right.$, $S \approx 90^{\circ}$ ), the coefficient of $\cos S$ in the matrix vanishes, and these stars do not contribute to the $\Delta \delta$ determination.

This method uses all of the stars, even those which were observed in only one zenith distance $(z)$. These stars do not contribute to the $\eta$ solution (equator correction) or the colour-magnitude effects, but they contribute to the $\Delta \delta$ determination and to the computed standard deviation.

The importance of this method is that it allows the utilization even of transit stars at only one zenith distance to get systematic differences $\Delta \delta$, which is not possible with the other two methods presented in this paper.

The method has the advantage to obtain simultaneously the differences in positions, the equator correction and the instrumental effects. The systematic effects are dependent of the star position and the physical characteristics that directly influence the quality of the observation. However, the system has a singularity, which is removed with the condition:

$\eta_{\mathrm{VL} 1}=\eta_{\mathrm{VL} 2}=\eta_{\mathrm{VL} 3}=\eta$

The results for latitude imply that $\eta$ is the same for all catalogues involved, and so the singularity of the system is removed.

\section{Results}

In this section, we show the results obtained by the three ways discussed in Sects. 2.1, 2.2 and 2.3.

\subsection{Results - Krejnin's method}

We used the same instrument to observe at two different zenith distances $\left(30^{\circ}\right.$ and $\left.45^{\circ}\right)$ at Valinhos. This should be quite effective in avoiding instrumental systematic differences. On the other hand, a single instrument obviously cannot make simultaneous observations in both zenith distances. As a consequence, latitude values $\phi_{A}$ and $\phi_{B}$ are themselves not simultaneous, and some interpolation scheme must be introduced for the utilization of Eq. (6).

\footnotetext{
1 Polynomial function: second degree to magnitude and first degree to colour index - (Boczko 1989; Martin \& Clauzet 1990; Martin et al. 1996).
} 
In the present results, we have computed the mean latitude with respect to the Bureau International de l'Heure (BIH/IERS) for both programmes.

The final computed values from Eq. (6) are:

$$
\begin{aligned}
& \eta_{A}-\eta_{B}=+0.007^{\prime \prime} \pm 0.010^{\prime \prime}(\mathrm{VL} 1 / \mathrm{VL} 3) \\
& \eta_{A}-\eta_{B}=-0.004^{\prime \prime} \pm 0.022^{\prime \prime}(\mathrm{VL} 2 / \mathrm{VL} 3)
\end{aligned}
$$

Before the application of Eq. (5), we must take into account instrumental problems affecting the computation of declinations. In fact the $\Delta \delta$ are obtained as an addition of the east and west residuals, which means that they may contain instrumental effects such as magnitude and colour index equations. To isolate these effects, we developed the term $C_{i}$ from Eq. (4) as a function of the magnitude and colour index for the maximum digression stars:

$$
C_{i}=B_{1} \cdot m_{N i}+B_{2} \cdot m_{N i}^{2}+A_{1} \cdot I_{N i}+\Delta z
$$

where the symbols $m_{N i}$ and $I_{N i}$ refer to normalized magnitude and normalized colour index for each star: $m_{N i}=$ $\left(m g_{i}-4\right) / 2$ and $I_{N i}=\left(I_{i}-0.8\right) / 1.2$, (Basso 1991).

The results obtained by a least squares method for VL1, VL2 and VL3 catalogues are showed in Table 1.

Table 1. Colour and magnitude coefficients for VL1, VL2 and VL3 catalogues - (maximum digression stars)

\begin{tabular}{llll}
\hline \multicolumn{3}{c}{ catalogues } \\
\hline & VL1 & VL2 & VL3 \\
\hline$B_{1}\left(^{\prime \prime}\right)$ & $+0.09 \pm 0.06$ & $-0.07 \pm 0.06$ & $+0.39 \pm 0.06$ \\
$B_{2}\left(^{\prime \prime}\right)$ & $+0.18 \pm 0.11$ & $+0.25 \pm 0.17$ & $+0.27 \pm 0.11$ \\
$A_{1}\left(^{\prime \prime}\right)$ & $-0.09 \pm 0.05$ & $-0.12 \pm 0.03$ & $-0.22 \pm 0.04$ \\
$\Delta z\left(^{\prime \prime}\right)$ & $-0.09 \pm 0.02$ & $-0.06 \pm 0.02$ & $-0.21 \pm 0.02$ \\
\hline & & (Martin & \& Clauzet 1990) \\
\hline
\end{tabular}

The larger values of VL3 than those in the other catalogues are probably due to the $45^{\circ}$ zenith distance observations. The high values confirm the importance of colour and magnitude equations in astrolabe data, as shown by Benevides-Soares (1988) and Chollet \& Sanchez (1990).

All declinations $\Delta \delta_{A i}^{\prime}$ and $\Delta \delta_{B i}^{\prime}$ from Eq. (5) were computed considering the colour and magnitude function above. After this we computed the values of $\eta_{A}$ and $\eta_{B}$ by means of Eqs. (5) and (6).

The results for VL1 and VL3 catalogues are:

$\eta_{A}=-0.04^{\prime \prime} \pm 0.02^{\prime \prime}$

$\eta_{B}=-0.04^{\prime \prime} \pm 0.02^{\prime \prime}$ and the results for VL2 and VL3 catalogues are:

$\eta_{A}=+0.06^{\prime \prime} \pm 0.17^{\prime \prime}$
$\eta_{B}=+0.07^{\prime \prime} \pm 0.17^{\prime \prime}$

These values, applied in Eq. (4), render the declinations absolute. It is important to note that, through the values of $\eta$, we can also extend the results to non-common stars.

\subsection{Results - method of differences}

The system represented by Eq. (7) was reduced for 29 common stars of VL1 $\left(30^{\circ}\right)$ and VL3 $\left(45^{\circ}\right)$ and 10 common stars of VL2 $\left(30^{\circ}\right)$ and VL3 $\left(45^{\circ}\right)$.

The values $C(m, I)$ for both catalogues are very similar to those obtained with the maximum digression stars presented in Table 1. These results are in agreement with those obtained by Benevides-Soares (1988), Boczko (1989) and Martin \& Clauzet (1990) with different procedures.

The equator corrections obtained by the Method of differences are:

a) for VL1 and VL3 catalogues:

$\eta=-0.06^{\prime \prime} \pm 0.04^{\prime \prime}$

$\zeta=+0.29^{\prime \prime} \pm 0.11^{\prime \prime}$ and

$\sigma_{\mathrm{G}}=0.47^{\prime \prime}$ (global standard deviation)

correlation $=0.25$

b) for VL2 and VL3 catalogues:

$\eta=+0.02^{\prime \prime} \pm 0.12^{\prime \prime}$

$\zeta=-0.10^{\prime \prime} \pm 0.19^{\prime \prime}$ and

$\sigma_{\mathrm{G}}=0.65^{\prime \prime}$ (global standard deviation)

correlation $=-0.26$.

The strong value of $\sigma_{\mathrm{G}}$ confirms the contamination by the colour and magnitude effects.

\subsection{Results - global reduction}

We used 381 different stars belonging to the VL1, VL2 and VL3 catalogues obtained at the OAM. Our goal was to determine 269 declination corrections $(\Delta \delta)$, taking into account that 112 stars were at maximum digression condition.

An analysis of the individual values of $\Delta \delta$ as obtained from global reduction, as well as the extension to the noncommon stars, was made based on FK5 system. The $\Delta \delta$ were obtained as sums of the east and west mean residuals, which means that they may contain instrumental effects such as magnitude and colour equations. In Table 2 , we have given the magnitude and colour index coefficients (instrumental effects) obtained by the global reduction that are comparable with the results obtained by Krejnin's method. 
Table 2. Colour and magnitude coefficients for VL1, VL2 and VL3 catalogues - (global reduction)

\begin{tabular}{llll}
\hline \multicolumn{3}{c}{ catalogues } \\
\hline & VL1 & VL2 & VL3 \\
\hline$B_{1}\left(^{\prime \prime}\right)$ & $+0.10 \pm 0.05$ & $-0.09 \pm 0.05$ & $+0.40 \pm 0.04$ \\
$B_{2}\left({ }^{\prime \prime}\right)$ & $+0.24 \pm 0.10$ & $+0.24 \pm 0.18$ & $+0.26 \pm 0.08$ \\
$A_{1}\left({ }^{\prime \prime}\right)$ & $-0.10 \pm 0.04$ & $-0.13 \pm 0.03$ & $-0.19 \pm 0.03$ \\
$\Delta z\left(^{\prime \prime}\right)$ & $-0.10 \pm 0.02$ & $-0.05 \pm 0.02$ & $-0.19 \pm 0.03$ \\
\hline & & & (Basso 1991) \\
\hline
\end{tabular}

These results are comparable with the best results presented in the literature. As we have seen in Table 1, the results for the VL3 catalogue are stronger and confirm the importance of colour and magnitude function in astrolabe data.

The general standard deviation obtained by the global reduction is $0.13^{\prime \prime}$, and the value of $\eta$ is $0.028^{\prime \prime} \pm 0.034^{\prime \prime}$ (Basso 1991).

Thus, the quality of the results is contaminated by the low quality of colour and magnitude errors.

The equator correction obtained is small, confirming the equator used in the FK5 system in the observed zone is compatible with the dynamical values for the equator correction taken with other techniques (Leister 1989), as shown in Fig. 1.

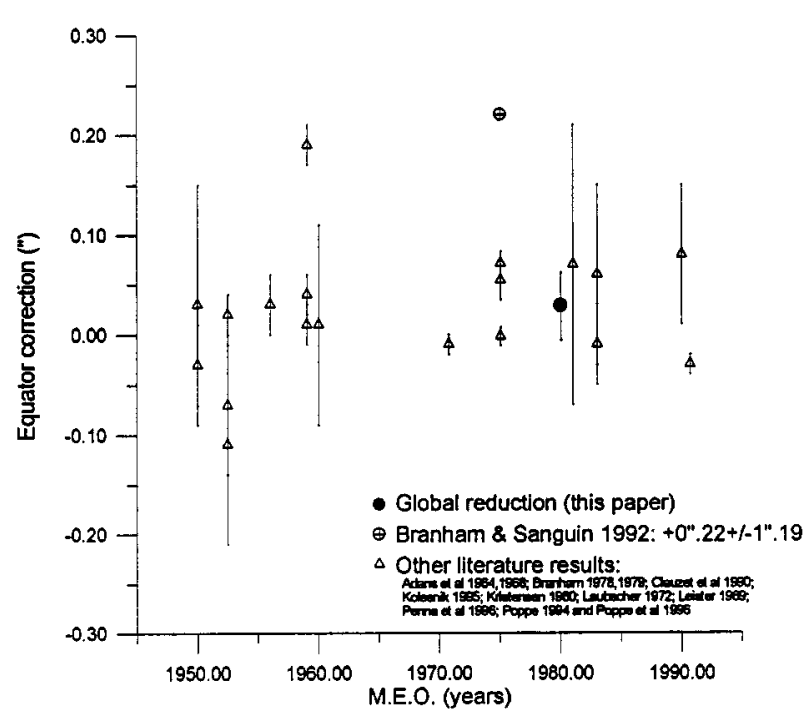

Fig. 1. Equator corrections

\section{Discussion}

In Table 3, we show the several equator corrections obtained from observations at Valinhos based on different selections of observational material. It seems that the better correction is that from our global reduction method.

It must be pointed out that, in our case, we estimated the equator correction and $\Delta \delta$ corrections for the declination zone of the astrolabe observations at Valinhos $\left(-68^{\circ} \leq \delta \leq+22^{\circ}\right.$, south hemisphere) shown in Fig. 2 .

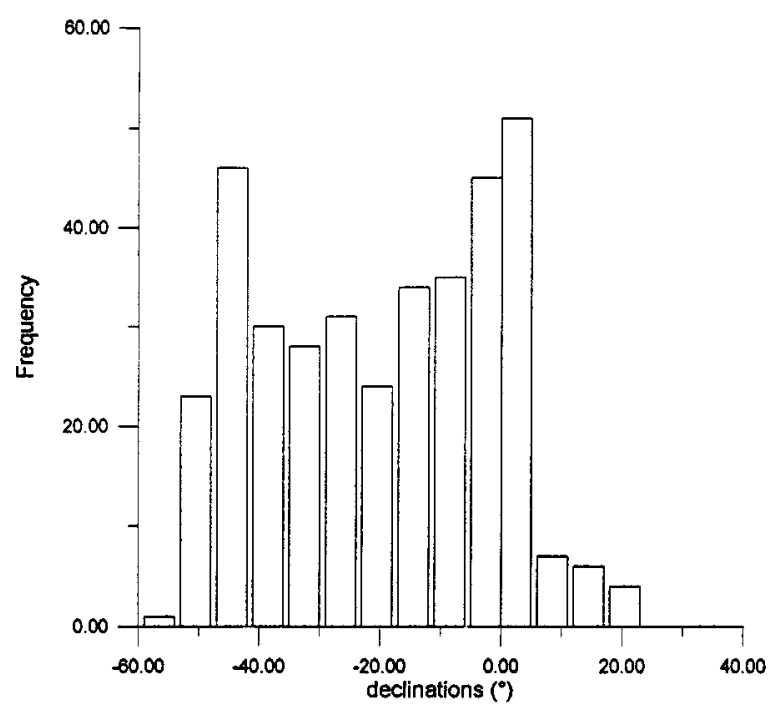

Fig. 2. The declination distribution of the data

We compare the absolute declinations obtained in this paper with the published (FK5-FK4) differences. There is a good correlation between these data (Fig. 3).

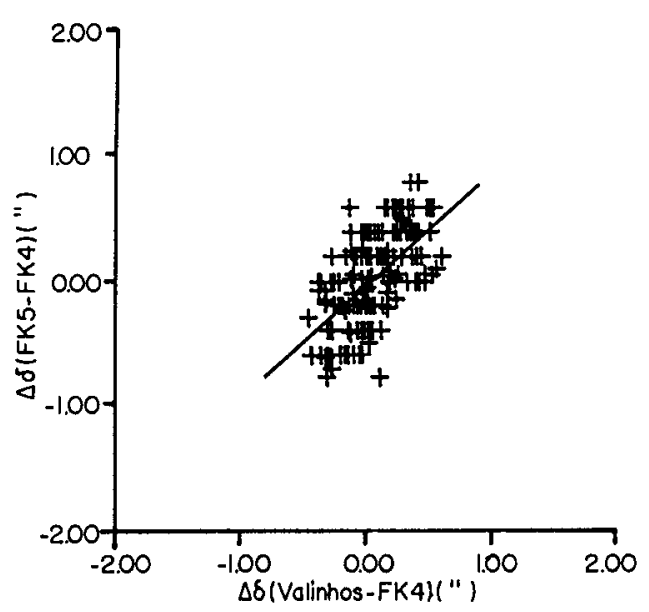

Fig. 3. Comparison of $\Delta \delta_{(\mathrm{OAM}-\mathrm{FK} 4)}$ with $\Delta \delta_{(\mathrm{FK} 5-\mathrm{FK} 4)}$ 
Table 3. Equator corrections

\begin{tabular}{lllll}
\hline Object & M.E.O. & $\eta\left(^{\prime \prime}\right)$ & $\sigma\left(^{\prime \prime}\right)$ & remarks \\
\hline Sun & 1980.9 & +0.07 & 0.14 & Leister (1989) - (OAM astrolabe data) \\
Sun & 1983.0 & -0.01 & 0.04 & Penna et al. (1996) - (OAM/CERGA/ON astrolabe data) \\
Stars & 1983.0 & +0.06 & 0.09 & Clauzet et al. (1990) - (OAM astrolabe data) \\
Sun & 1990.0 & +0.08 & 0.07 & Poppe (1994) - (OAM/CERGA astrolabe data) \\
Sun & 1990.7 & -0.03 & 0.01 & Poppe et al. (1996) - (OAM/CERGA astrolabe data) \\
& & & & \\
This paper & 1980.7 & -0.04 & 0.02 & Krejnin's Method \\
(Stars) & & -0.04 & 0.02 & Krejnin's Method \\
& & +0.06 & 0.17 & Krejnin's Method \\
& & +0.07 & 0.17 & Krejnin's Method \\
& & -0.06 & 0.04 & Method of Differences \\
& +0.02 & 0.12 & Method of Differences \\
& & & \\
& & & & \\
& & &
\end{tabular}

The analysis of Fig. 3 confirms that the results are in agreement with the published values and that the Valinhos catalogues can represent corrections to the FK4 catalogue in the same sense that the FK5 does.

\section{Conclusions}

Despite the small number of common stars in the VL1, VL2 and VL3 catalogues, the results confirm the opportunity and validity of the global reduction to compute absolute declinations. The comparison of our results with the dynamical ones confirms the astrolabe as a potential instrument for ground-based astrometry.

Table 3 gives the equator corrections obtained by Leister (1989), Penna et al. (1996), Clauzet et al. (1990), Poppe (1994), Poppe et al. (1996), and also the results obtained in this paper. These values are in agreement with one another. This is due the fact that the results obtained with the Sun uses the same set of stars.

Thus, the astrolabe can be a very important instrument for the definition of the fundamental reference equator. The results confirm also the position adopted by Fricke (1982) in order to maintain the equator of the FK4 system in the FK5 system.

A special observational programme made at two zenith distances (VL4 \& VL5), including more than one hundred common stars, is underway at the OAM. We hope that this programme will establish, once and for all, the usefulness of equal altitude observations in deriving absolute declinations. Thus, the astrolabe can be used as a producer of stellar catalogues.

Acknowledgements. The authors would like to thank Wilson Monteiro for most of the observations taken in this observational programme. V.A.F. Martin is also grateful to Dr. L.B.F. Clauzet (in memorium) for his incentive and several aids at the beginning of her job in astrometry and also to Dr. P. BenevidesSoares for his help in the global reduction method. Financial support from CNPq, FAPESP, CAPES and "Fundo Bunka de Pesquisa/1993" are gratefully acknowledged.

\section{References}

Adans A.N., Bestul S.M., Scott D.K., 1964, Publ. U. S. Naval Obs. 19 , pt. 1

Adans A.N., Scott D.K., 1968, Publ. U. S. Naval Obs. 19, pts. 1 and 2

Basso V.A.F., 1991, MSc. Thesis, IAGUSP, Brazil

Benevides-Soares P., Clauzet L.B.F., 1986, in: IAU Symp. 109, Astrometric Techniques, Eichhorn H.K., Leacock R.J. (eds.). Reidel Dordrecht, p. 103

Benevides-Soares P., 1988, A\&A 189, 297-302

Benevides-Soares P., Teixeira R., 1992, A\&A 253, 307-310

Boczko R., 1989, PhD. Thesis, IAGUSP, Brazil

Branham Jr. R.L., 1978, AJ 83, 675-681

Branham Jr. R.L., 1979, AJ 84, 1399-1401

Branham Jr. R.L., Sanguin J.G., 1992, AJ 103, 2099

Chollet F., Sanchez M., 1990, A\&A 234, 576-582

Chollet F., Najid N.-E., 1992, A\&A 262, 341-346

Chollet F., 1993, A\&A 280, 675-682

Clauzet L.B.F., 1983, A\&AS 52, 403-410

Clauzet L.B.F., Benevides-Soares P., 1985, A\&AS 61, 83-88

Clauzet L.B.F., 1987, These de Livre Docência, IAGUSP, Brazil

Clauzet L.B.F., 1989, A\&AS 77, 67-72

Clauzet L.B.F., Benevides-Soares P., Leister N.V., Martin V.A.F., 1990, in: Colloque Andre Danjon - Journeés 1990 - Systèmes de Référence Spatio-Temporels, Capitaine N. (ed.) Paris, p. 89

Débarbat S., Guinot B., 1970, La méthode des hauteurs égales en astronomie. Gordon \& Breach, Paris

Eichhorn H.K., 1960, Astron. Nachr. 285, 233

van Flandern T.C., 1971a, Celest. Mech. 4, 182

van Flandern T.C., 1971b, AJ 76, 81-82 
Fricke W., 1978, in: Methods of compiling a Fundamental Reference Systems - Colloquium on European Satellite Astrometry, Padova, p. 175

Fricke W., 1982, A\&A 107, L13-L14

Kolesnik Y.B., 1995, A\&A 294, 874-894

Krejnin E.I., 1968, AZh 45, 447-451

Krejnin E.I., 1986, SvA 30, No. 3

Kristensen L.K., 1980, Mitteil. Astron. Gesellschaft. 48

Laubscher R.E., 1972, A\&A 20, 407-414

Leister N.V., 1989, PhD. Thesis, IAGUSP, Brazil

Martin V.A.F., Clauzet L.B.F., 1990, Rev. Mex. Astron.
Astrof. 21, 297-299

Martin V.A.F., Boczko R., Benevides-Soares P., Leister N.V., 1996, A\&AS 118, 525-528

Najid N.-E., 1993, A\&AS 102, 389-396

Penna J.L., Benevides-Soares P., Andrei A.H., Laclare F., Leister N.V., 1996, A\&A 310, 1036

Podobed V.V., 1965, Fundamental Astrometry. The University of Chicago Press, EUA

Poppe P.C.R., 1994, MSc. Thesis, IAGUSP, Brazil

Poppe P.C.R., Leister N.V., Laclare F., 1996, A\&A 315, 633 